\title{
Association of pulse rate with outcomes in heart failure with reduced ejection fraction: a retrospective cohort study
}

Katherine E. Kurgansky ${ }^{1}$, Petra Schubert ${ }^{1}$, Rachel Parker ${ }^{1}$, Luc Djousse ${ }^{1,2}$, Jerome B. Riebman ${ }^{3}$,

David R. Gagnon ${ }^{1,4 \dagger}$ and Jacob Joseph $h^{1,5,6^{*}+}$ (D)

\begin{abstract}
Background: In a real-world setting, the effect of pulse rate measured at the time of diagnosis and serially during follow-up and management, on outcomes in heart failure with reduced ejection fraction (HFrEF), has not been wellstudied. Furthermore, how beta-blockade use in a real-world situation modifies this relation between pulse rate and outcomes in HFrEF is not well-known. Hence, we identified a large, national, real-world cohort of HFrEF to examine the association of pulse rate and outcomes.

Methods: Using Veterans Affairs (VA) national electronic health records we identified incident HFrEF cases between 2006 and 2012. We examined the associations of both baseline and serially measured pulse rates, with mortality and days hospitalized per year for heart failure and for any cause, using crude and multivariable Cox proportional hazards and Poisson or negative binomial models, respectively. The exposure was examined as continuous, dichotomous, and categorical. Post-hoc analyses addressed the interaction of pulse rate and beta-blocker target dose.

Results: We identified 51,194 incident HFrEF cases (67 \pm 12 years, 98\% male, 77\% white. A significant positive, near linear relationship was observed for both baseline and serially measured pulse rates with all-cause mortality, allcause hospitalization and heart failure hospitalization after adjusting for covariates including beta-blocker use. Patients who had a pulse rate $\geq 70$ bpm in the past 6 months had 36\% (95\% Cl: 31-42\%), 25\% (95\% Cl: 19-32\%), and 51\% (95\% Cl: 33-72\%) increased rates of mortality, all-cause hospitalization, and heart failure hospitalization, respectively, compared to patients with pulse rates $<70 \mathrm{bpm}$. A minority of subjects (15\%) were treated with guideline directed beta blockade $\geq 50 \%$ of recommended target dose, among whom better outcomes were seen compared to those who did not achieve target dose in patients with pulse rates both above and below 70 beats per minute.

Conclusions: High pulse rate, both at the time of diagnosis and during follow-up, is strongly associated with increased risk of adverse outcomes in HFrEF patients, independent of the use of beta-blockers. In a real-world setting, the majority of HFrEF patients do not achieve target dose of beta-blockade; greater use of strategies to reduce heart rate may improve outcomes in HFrEF.
\end{abstract}

Keywords: Heart failure, Heart rate, Outcomes, Beta-blocker, Hospitalization, Mortality

\footnotetext{
* Correspondence: jjoseph16@partners.org

${ }^{\dagger}$ David R. Gagnon and Jacob Joseph are senior authors

'Massachusetts Veterans Epidemiology and Research Information Center

(MAVERIC), Veterans Affairs Boston Healthcare System, Boston, MA, USA

${ }^{5}$ Department of Medicine, Division of Cardiovascular Medicine, Brigham and

Women's Hospital, Harvard Medical School, Boston, MA, USA

Full list of author information is available at the end of the article
}

(c) The Author(s). 2020 Open Access This article is distributed under the terms of the Creative Commons Attribution 4.0 International License (http://creativecommons.org/licenses/by/4.0/), which permits unrestricted use, distribution, and reproduction in any medium, provided you give appropriate credit to the original author(s) and the source, provide a link to the Creative Commons license, and indicate if changes were made. The Creative Commons Public Domain Dedication waiver (http://creativecommons.org/publicdomain/zero/1.0/) applies to the data made available in this article, unless otherwise stated. 


\section{Background}

Despite significant advances in care, HFrEF continues to have high rates of mortality and morbidity $[1,2]$. Studies have shown that high resting heart rate is an independent risk factor for all-cause mortality, cardiovascular mortality, and cardiovascular events in the general population [3, 4], as well as in those with cardiovascular disease [5-7], coronary artery disease [8-10], hypertension $[7,11]$, heart failure [12], and diabetes [13]. The relation of heart rate to adverse outcomes may be mediated via its effects on coronary blood flow, cardiac contractility, and energy expenditure $[5,14]$. These findings suggest that heart rate reduction may be an important target for clinicians to improve disease outcomes. A randomized controlled trial conducted in European patients with HFrEF, the Ivabradine and outcomes in Chronic Heart Failure (SHIFT) study, demonstrated that reduction of heart rate utilizing ivabradine is beneficial in HFrEF with heart rates $>70 \mathrm{bpm}$ in spite of guideline directed therapy including beta blockers [15]. However, the relation of heart rate to outcomes in a real-world situation has not been well evaluated.

We conducted an observational study with long-term follow-up using national electronic health record data to examine the hypothesis that pulse rate (as an equivalent measure of heart rate), measured both around the time of diagnosis and repeatedly over time, is associated with mortality and hospitalization outcomes in HFrEF patients. Our results suggest that there is a strong, positive linear association between baseline, as well as serially measured pulse rate and risk of mortality and hospitalization outcomes, especially at values above 70 bpm.

\section{Methods}

\section{Cohort selection}

We identified incident cases of HFrEF diagnosed between $1 / 1 / 2006$ and $12 / 31 / 2012$ in the national Veterans Affairs (VA) healthcare system. Cases were defined as having an International Classification of Disease ninth revision (ICD-9) code for the diagnosis of heart failure (428.xx) and a left ventricular ejection fraction (LVEF) $\leq 35 \%$. The date of the first occurrence of LVEF $\leq 35 \%$ was defined as the index date. To ensure these were incident cases, any patients who had a heart failure diagnosis code 1 year prior until 30 days before the index date in VA or in Centers for Medicare \& Medicaid Services (CMS) data [16] were excluded. We excluded patients who had an atrial fibrillation or atrial flutter diagnosis prior to the index date not only to ensure presence of sinus rhythm, but also to ensure that pulse rate was equivalent to heart rate. To ensure sinus rhythm, we also excluded those who ever had a heart transplant or had paced rhythm within 6 months prior to the index date.

\section{Exposure}

Baseline pulse rate (within 10 days after index date, outpatient or last inpatient value) and serially recorded time-varying pulse-rate, updated at 6-month intervals following the index date, were the exposures studied. For serial values, the first pulse rate measurement was recorded within the 6-month period after the index date with outcomes assessed from 6 through 12 months. Successive pulse values fell within the 6 months before the start of each subsequent 6-month window during which outcomes were assessed. Individual pulse rates $<30$ or $>$ $180 \mathrm{bpm}$ were excluded. Pulse rates were considered equivalent to heart rate since they are generally verified by a physician or physician extender and corrected if there is a discrepancy between pulse rate measured by clinic staff and heart rate measured by clinical provider.

\section{Outcomes}

The outcomes analyzed were all-cause mortality, and number of days hospitalized per year for any cause or for heart failure. Patients were followed in the VA databases supplemented by CMS data [16] from 6 months post index date for the longitudinal analyses and from the time of baseline pulse measurement for the baseline analyses. For both analyses, follow-up ended at death, last VA visit, or $12 / 31 / 2013$. For the longitudinal analyses, outcomes were assessed within each 6-month interval following the 6-month interval in which pulse rate was measured. Patients with any inpatient stay longer than 180 days were excluded from the hospitalization outcome analyses.

\section{Covariates}

Demographic, anthropometric, comorbidity, and laboratory variables were extracted from the VA healthcare system database. For the longitudinal analyses, timevarying covariates were updated at each 6-month interval with values that fell in the prior interval, closest to the start of the current interval. For the baseline analyses, covariates were selected within 30 days before and closest to the date of the baseline pulse rate measurement.

Prescription information for medication use was obtained from the pharmacy records within the VA healthcare system database and CMS [16]. We considered someone a user if they had a prescription dispensed within 6 months prior to the date of baseline pulse rate or the interval start date. We also categorized beta blocker use into $<50 \%$ target dose and $\geq 50 \%$ target dose, based on the type of beta blocker and dose at 1 year after diagnosis. Target doses were calculated for carvedilol, 
metoprolol succinate, and bisoprolol according to established treatment guidelines [17]; target doses were not analyzed for other beta blockers. Additional information regarding cohort selection, exposure, and covariates can be found in the supplementary methods.

\section{Statistical analysis}

To evaluate pulse rate as a continuous predictor of allcause mortality and hospitalization outcomes, we fit Cox proportional hazards and Poisson or negative binomial regression cubic spline plots, respectively. Generalized estimating equation (GEE) based negative binomial regression models with an ar (1) correlation structure were fit for hospitalization outcomes with longitudinal exposures, while Poisson models were fit for the baseline exposures. We used SAS macros to create natural cubic spline plots, specifying 5-7 knots equally distributed across the data, 3 degrees of freedom, and a reference value of $75 \mathrm{bpm}$.

Based on the relationship observed in the cubic spline plots, we categorized both repeated and baseline pulse rates by deciles: $47-59 \mathrm{bpm}, 60-63 \mathrm{bpm}, 64-67 \mathrm{bpm}$, 68-71 bpm, 72-75 bpm, 76-79 bpm, 80-83 bpm, 84-88 bpm, 89-96 bpm, and $97-117 \mathrm{bpm}$. Pulse rates that fell below the 1st percentile $(47 \mathrm{bpm})$ and above the 99th percentile $(117 \mathrm{bpm})$ were excluded. We selected $72-75$ $\mathrm{bpm}$ as the reference group since the median and mean pulse values fell within that range and it is considered clinically normal. Additionally, we categorized elevated $(\geq 70 \mathrm{bpm})$ and low pulse values $(<70 \mathrm{bpm})$. These decile and dichotomous categories were used for all proceeding regression analyses.

Regression models were built, starting with crude, then age-adjusted, parsimonious (age, gender, race), and finally multivariable models adjusted for age, gender, race, body mass index (BMI), estimated glomerular filtration rate (eGFR), serum potassium, serum sodium, use of angiotensin receptor blockers (ARBs), angiotensin converting enzyme inhibitors (ACEIs), nitrates, statins, aldosterone antagonists, calcium channel blockers, loop diuretics, and beta blockers, and history of coronary artery disease, hypertension, hyperlipidemia, chronic obstructive pulmonary disease, dementia, type II diabetes, stroke/ transient ischemic attack (TIA), cardiovascular disease, and anemia. Baseline multivariable models also adjusted for baseline LVEF and longitudinal analyses adjusted for development of atrial fibrillation or atrial flutter.

For both baseline and longitudinal analyses, Cox proportional hazards regression models were fit to examine the association between pulse rate category and all-cause mortality. Using a counting process approach [18], subjects entered the model at the time of their baseline pulse rate or the beginning of the 6- month interval, and person-time accrued until the end of the interval (for longitudinal analysis), death, last VA visit, or the end of follow-up (12/31/2013). Through examination of hazard and $\log$ negative $\log$ plots we concluded that the proportional hazards assumption was met. Hazard ratios (HR) and 95\% confidence intervals (CI) were estimated for all pulse categories relative to the reference group.

GEE-based negative binomial regression models with an ar (1) correlation structure were fit to examine the association between longitudinal pulse rate categories and number of all-cause and heart failure hospitalization days per year. Person-observation-years were counted as the time (in years) that each subject was observed within each 6-month interval. Each subject could contribute to multiple pulse rate categories as their pulse values changed across 6-month intervals. The number of person-observation-years (on the $\log$ scale) was included as an offset in the models. Poisson regression models were fit to examine the association between baseline pulse rate and number of all-cause and heart failure hospitalization days per year, with years observed (on the log scale) included as an offset in the models. Rate ratios (RR) and 95\% confidence intervals were estimated for all pulse rate categories relative to the reference group.

Our post-hoc sub-analysis included mortality cumulative incidence plots derived from multivariable adjusted Cox proportional hazards models stratified by pulse rate category to examine how survival varied by the interaction of beta blocker target dose $\geq$ and $<50 \%$ and pulse rate $\geq$ and $<70 \mathrm{bpm}$.

All analyses were conducted using SAS Enterprise Guide 7.1 (SAS institute Inc., Cary, NC). P-values are 2tailed and significant at the alpha $=0.05$ level.

\section{Results}

As shown in Fig. 1, the longitudinal analysis cohort (main cohort) and baseline analysis cohorts (subset that had baseline pulse rate values) consisted of 51,194 and 34,402 patients respectively. In the longitudinal analysis cohort, mean age was $67 \pm 12$ years, $98 \%$ were male, and $77 \%$ were white, as expected in a veteran population (Table 1). At the first 6-month time point, those in the lowest decile $(47-59 \mathrm{bpm})$ were older, less likely to be African American, had lower eGFR, lower prevalence of COPD and diabetes, and higher prevalence of CAD and hyperlipidemia than those in the highest decile group (97-117 bpm). Similar patterns were seen across deciles for laboratory measurements and comorbidities at the 1.5-year timepoint (data not shown).

As shown in Table 2, within the first 6 months after the index date, $81 \%$ were on a beta blocker, $77 \%$ on an ACEI or ARB, and $16 \%$ on aldosterone antagonists. 


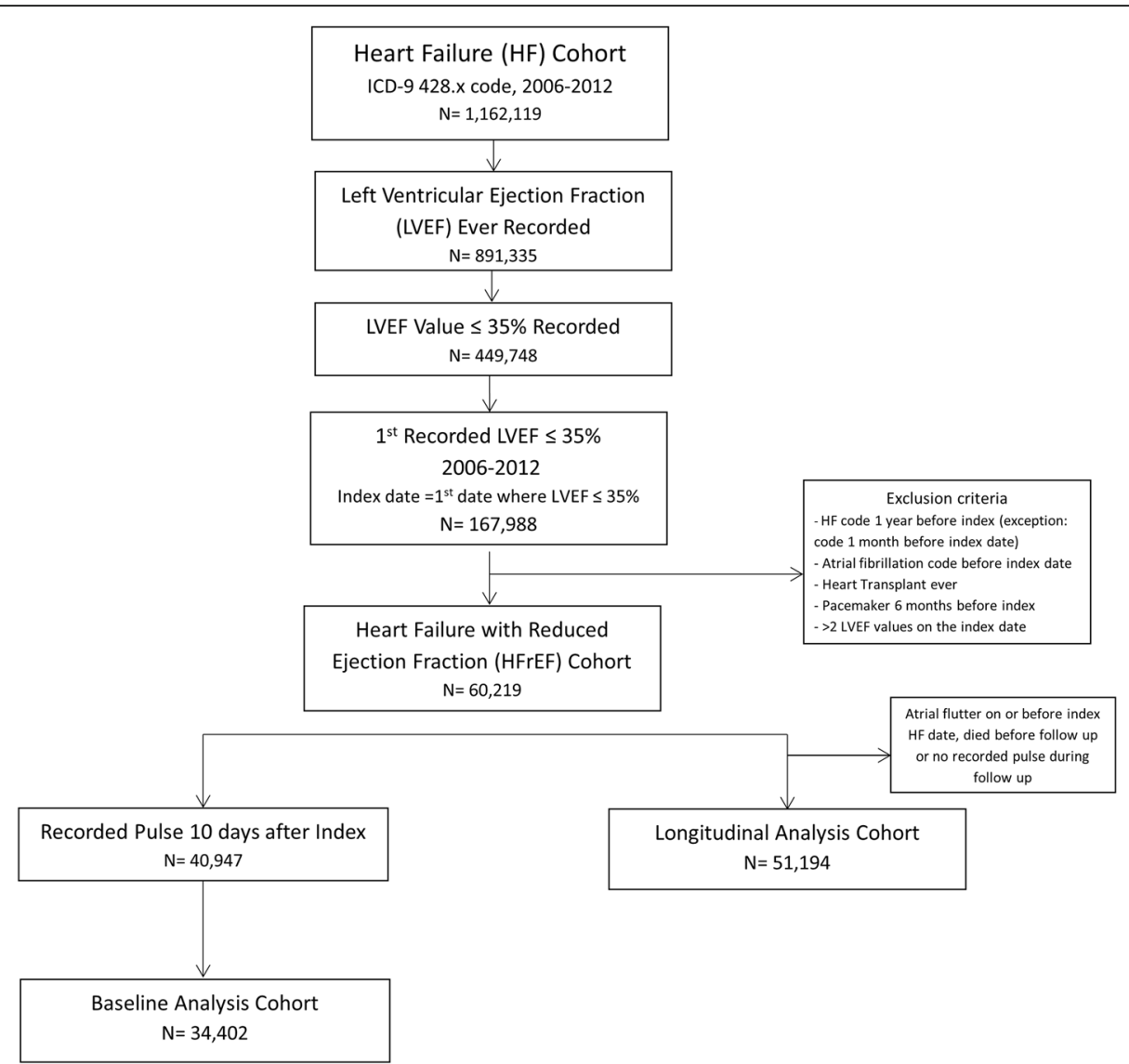

Fig. 1 Flow chart for creation of Heart Failure with Reduced Ejection Fraction (HFrEF) cohorts

However, by 1.5 years the rates of use dropped to $73 \%$ for beta blocker, $67 \%$ for ACEI or ARB, and $15 \%$ for aldosterone antagonists. During the first 6 months after diagnosis, compared to the highest decile, patients in the lowest decile had higher use of beta blockers, statins, and nitrates, and lower use of loop diuretics. Use of ACEI or ARB varied less across deciles. Similar trends were seen across pulse rate deciles at 1.5 years for beta blocker, statin, and nitrate use. For many medications, the greatest decline in use between intervals was in the highest decile. No patients in our study were treated with ivabradine over the course of follow-up. Only $12.3 \%$ of patients received implantable cardioverter defibrillators (ICD) and only $4.2 \%$ underwent cardiac resynchronization therapy (CRT) following HFrEF diagnosis prior to the end of study follow-up.

\section{Associations of baseline pulse rate with outcomes}

As shown in Fig. 2a-c and Table 3, baseline pulse rate demonstrated significant positive, near linear associations with mortality and hospitalization outcomes. When comparing patients with baseline pulse rate $\geq 70$ versus $<70$, the following hazard and rate ratios were obtained: all-cause mortality - 1.26 (95\% CI: 1.20-1.33); all-cause hospitalizations - 1.20 (95\% CI: 1.12-1.28); and heart failure hospitalizations - 1.50 (95\% CI: 1.27-1.77). Associations were significant in more pulse rate deciles for all-cause mortality and all-cause hospitalizations compared to heart failure hospitalizations (an outcome with lower event rates).

\section{Association of longitudinal pulse rate with all-cause mortality}

Over a median follow-up of 3.2 years (IQR: 1.6-5.1) in the longitudinal analysis cohort, 16,370 deaths occurred; during the first 6 months after heart failure diagnosis, an additional 3844 patients died who were not included in the analysis, as follow-up started at 6 months.

As shown in Fig. 2d, repeated pulse rate measures demonstrated a positive linear relationship with allcause mortality above a pulse rate of around $70 \mathrm{bpm}$. When comparing patients who had a pulse rate $\geq 70$ in the prior 6-month interval versus those with a pulse rate $<70$, we observed a hazard ratio of $1.36(95 \% \mathrm{CI}$ : 1.31-1.42) for all-cause mortality. Table 3 presents the association of repeated pulse rate measurements categorized into deciles with all outcomes. 
Table 1 Patient characteristics in first six-month interval after HFrEF diagnosis

\begin{tabular}{|c|c|c|c|c|c|}
\hline Characteristics & $\begin{array}{l}\text { Overall } \\
N=51,194\end{array}$ & $\begin{array}{l}\text { 1st Pulse Rate Decile } \\
\text { (47-59 bpm) } \\
n=7026\end{array}$ & $\begin{array}{l}\text { 5th Pulse Rate Decile } \\
\text { (72-75 bpm) } \\
n=5479\end{array}$ & $\begin{array}{l}\text { 10th Pulse Rate Decile } \\
\text { (97-117 bpm) } \\
n=2929\end{array}$ & $\begin{array}{l}p- \\
\text { value }\end{array}$ \\
\hline Age, y & $66.9 \pm 11.5$ & $69.4 \pm 11$ & $11.4 \pm 62.5$ & $62.5 \pm 10.9$ & $<.0001$ \\
\hline Sex (male), $\%$ & 50,267 (98.2\%) & $6949(98.9 \%)$ & $5378(98.2 \%)$ & $2841(97.0 \%)$ & $<.0001$ \\
\hline \multicolumn{6}{|l|}{ Race, $\%$} \\
\hline White & 35,577 (76.9\%) & $5061(79.7 \%)$ & $3782(76.4 \%)$ & $1833(68.5 \%)$ & $<.0001$ \\
\hline African American & $9784(21.1 \%)$ & $1170(18.4 \%)$ & $1062(21.5 \%)$ & $798(29.8 \%)$ & \\
\hline Other & $912(2.0 \%)$ & $122(1.9 \%)$ & $106(2.1 \%)$ & $46(1.7 \%)$ & \\
\hline \multicolumn{6}{|l|}{$\mathrm{BMI}, \%$} \\
\hline$<18.5, \mathrm{~kg} / \mathrm{m}^{2}$ & 1225 (2.4\%) & $131(1.9 \%)$ & $123(2.3 \%)$ & $137(4.8 \%)$ & $<.0001$ \\
\hline $18.5-24.9, \mathrm{~kg} / \mathrm{m}^{2}$ & 12,766 (25.7\%) & $1959(28.6 \%)$ & $1360(25.5 \%)$ & $807(28.2 \%)$ & \\
\hline $25-29.9, \mathrm{~kg} / \mathrm{m}^{2}$ & $16,551(33.3 \%)$ & $2413(35.2 \%)$ & $1717(32.2 \%)$ & $816(28.5 \%)$ & \\
\hline $30-34.9, \mathrm{~kg} / \mathrm{m}^{2}$ & $10,998(22.1 \%)$ & $1425(20.8 \%)$ & $1221(22.9 \%)$ & $580(20.3 \%)$ & \\
\hline$\geq 35, \mathrm{~kg} / \mathrm{m}^{2}$ & $8203(16.5 \%)$ & $929(13.6 \%)$ & $915(17.2 \%)$ & $520(18.2 \%)$ & \\
\hline Baseline EF, \% & $26.6 \pm 7.4$ & $26.9 \pm 7.4$ & $26.6 \pm 7.3$ & $25.8 \pm 7.5$ & $<.0001$ \\
\hline Serum Potassium, mEq/L & $4.3 \pm 0.5$ & $4.4 \pm 0.5$ & $4.3 \pm 0.5$ & $4.3 \pm 0.5$ & $<.0001$ \\
\hline Serum Sodium, mmol/L & $138.9 \pm 3.2$ & $139.2 \pm 2.9$ & $138.9 \pm 3.1$ & $138.2 \pm 3.3$ & $<.0001$ \\
\hline eGFR, $\mathrm{mL} / \mathrm{min} / 1.73 \mathrm{~m}^{2}$ & $67.7 \pm 24.1$ & $63.7 \pm 22.9$ & $67.5 \pm 24$ & $74.9 \pm 25.6$ & $<.0001$ \\
\hline Anemia, \% & 24,707 (48.3\%) & $3591(51.1 \%)$ & $2693(49.2 \%)$ & $1591(54.3 \%)$ & 0.0001 \\
\hline Coronary artery disease, $\%$ & $37,147(72.6 \%)$ & $5484(78.1 \%)$ & $4097(74.8 \%)$ & $1820(62.1 \%)$ & $<.0001$ \\
\hline Chronic obstructive pulmonary disease, $\%$ & $17,902(35.0 \%)$ & $2286(32.5 \%)$ & $1926(35.2 \%)$ & $1409(48.1 \%)$ & $<.0001$ \\
\hline Dementia, \% & $5944(11.6 \%)$ & $865(12.3 \%)$ & $625(11.4 \%)$ & $493(16.8 \%)$ & $<.0001$ \\
\hline Diabetes, \% & $25,011(48.9 \%)$ & $3155(44.9 \%)$ & $2839(51.8 \%)$ & $1596(54.5 \%)$ & $<.0001$ \\
\hline Hypertension, \% & $42,866(83.7 \%)$ & $6155(87.6 \%)$ & $4671(85.3 \%)$ & $2483(84.8 \%)$ & $<.0001$ \\
\hline Peripheral vascular disease, $\%$ & $13,091(25.6 \%)$ & $1981(28.2 \%)$ & $1423(26.0 \%)$ & $760(26.0 \%)$ & 0.0025 \\
\hline Stroke/Transient ischemic attack, \% & $9494(18.6 \%)$ & $1547(22.0 \%)$ & $1025(18.7 \%)$ & $512(17.5 \%)$ & $<.0001$ \\
\hline Hyperlipidemia, \% & $38,370(75.0 \%)$ & $5577(79.4 \%)$ & $4229(77.2 \%)$ & $2006(68.5 \%)$ & $<.0001$ \\
\hline Atrial fibrillation/flutter, \% & $2673(5.2 \%)$ & $464(6.6 \%)$ & $301(5.5 \%)$ & 188 (6.4\%) & $<.0001$ \\
\hline
\end{tabular}

\section{Associations of longitudinal pulse rate with all-cause hospitalizations}

As shown in Fig. 2e and Table 3, a significant, positive linear association was observed between repeated pulse rate measurements $\geq 80 \mathrm{bpm}$ and number of days allcause hospitalization per year. Patients who had a pulse rate $\geq 70 \mathrm{bpm}$ in the past 6 months had 1.25 times $(95 \%$ CI: 1.19-1.32) increased rate of hospitalizations compared to those with pulse rate $<70 \mathrm{bpm}$.

\section{Associations of longitudinal pulse rate with heart failure hospitalizations}

A positive, linear relationship was demonstrated for repeated pulse rate measurements $\geq 80 \mathrm{bpm}$ and number of days hospitalized for heart failure per year (Fig. $2 \mathrm{f}$ and Table 3), with statistically significant increases noted in the 9th and 10th deciles. Patients who had a pulse rate $\geq$ $70 \mathrm{bpm}$ in the past 6 months had 1.51 times (95\% CI:
1.33-1.72) increased rate of hospitalizations for heart failure per year compared to those with pulse rate $<70$ bpm.

\section{Effect of Beta blocker dose}

Only 19,453 patients were on guideline recommended beta blockers, of whom only 7915 ( $15 \%$ of total cohort) were on $\geq 50 \%$ target doses. Figure 3 demonstrates the cumulative incidence of all-cause mortality by beta blocker dose ( $\geq$ or $<50 \%$ target dose) for patients with pulse rate $<70$ and $\geq 70 \mathrm{bpm}$ at 1 year after the index date. Among patients with pulse rate $<70 \mathrm{bpm}$, those who achieved $\geq 50 \%$ beta blocker target dose had significantly improved mortality outcomes than those at $<50 \%$ beta blocker target dose $(p=0.0113)$. Similarly, among patients with pulse rate $\geq 70 \mathrm{bpm}$, those who achieved $\geq 50 \%$ beta blocker target dose had significantly better 
Table 2 Medication Use in Intervals 1 (6 months after diagnosis) and 3 (1-1.5 years after diagnosis)

\begin{tabular}{|c|c|c|c|c|c|c|c|c|c|c|}
\hline \multirow{4}{*}{ Characteristics } & \multicolumn{5}{|c|}{ Interval 1 (First 6 months) } & \multicolumn{5}{|c|}{ Interval 3 ( $1-1.5$ years) } \\
\hline & Overall & $\begin{array}{l}\text { 1st Pulse } \\
\text { Rate Decile }\end{array}$ & $\begin{array}{l}\text { 5th Pulse } \\
\text { Rate Decile }\end{array}$ & $\begin{array}{l}\text { 10th Pulse } \\
\text { Rate Decile }\end{array}$ & $\begin{array}{l}p- \\
\text { value }\end{array}$ & Overall & $\begin{array}{l}\text { 1st Pulse } \\
\text { Rate Decile }\end{array}$ & $\begin{array}{l}\text { 5th Pulse } \\
\text { Rate Decile }\end{array}$ & $\begin{array}{l}\text { 10th Pulse } \\
\text { Rate Decile }\end{array}$ & $\begin{array}{l}p- \\
\text { value }\end{array}$ \\
\hline & $\begin{array}{l}N=44 \\
093\end{array}$ & $(47-59 \mathrm{bpm})$ & (72-75 bpm) & (97-117 bpm) & & $\begin{array}{l}N=44 \\
093\end{array}$ & (47-59 bpm) & (72-75 bpm) & (97-117 bpm) & \\
\hline & & $n=6116$ & $n=4753$ & $n=2269$ & & & $n=6433$ & $n=5094$ & $n=2214$ & \\
\hline $\begin{array}{l}\text { ACE Inhibitor or } \\
\text { ARB, } \%\end{array}$ & $\begin{array}{l}31,716 \\
(77.2 \%)\end{array}$ & $4808(78.6 \%)$ & $3626(76.3 \%)$ & $1741(76.7 \%)$ & 0.0007 & $\begin{array}{l}28,970 \\
(67.0 \%)\end{array}$ & $4523(70.3 \%)$ & $3433(67.4 \%)$ & $1349(60.9 \%)$ & $<.0001$ \\
\hline $\begin{array}{l}\text { Aldosterone } \\
\text { Antagonists, \% }\end{array}$ & $\begin{array}{l}6574 \\
(16.0 \%)\end{array}$ & $878(14.4 \%)$ & 789 (16.6\%) & $410(18.1 \%)$ & $<.0001$ & $\begin{array}{l}6374 \\
(14.8 \%)\end{array}$ & $838(13.0 \%)$ & 778 (15.3\%) & $308(13.9 \%)$ & $<.0001$ \\
\hline Beta blocker, \% & $\begin{array}{l}33,407 \\
(81.3 \%)\end{array}$ & $5177(84.6 \%)$ & $\begin{array}{l}3861 \\
(81.20 \%)\end{array}$ & $1696(74.7 \%)$ & $<.0001$ & $\begin{array}{l}31,735 \\
(73.4 \%)\end{array}$ & $5031(78.2 \%)$ & $3804(74.7 \%)$ & $1373(62.0 \%)$ & $<.0001$ \\
\hline $\begin{array}{l}\text { Calcium channel } \\
\text { blocker, \% }\end{array}$ & $\begin{array}{l}8626 \\
(21.0 \%)\end{array}$ & $1536(25.1 \%)$ & 945 (19.9\%) & $404(17.8 \%)$ & $<.0001$ & $\begin{array}{l}7861 \\
(18.2 \%)\end{array}$ & $1442(22.4 \%)$ & 890 (17.5\%) & $353(15.9 \%)$ & $<.0001$ \\
\hline Statins, $\%$ & $\begin{array}{l}29,996 \\
(73.00 \%)\end{array}$ & $4644(75.9 \%)$ & $\begin{array}{l}3488 \\
(723.4 \%)\end{array}$ & $1480(65.2 \%)$ & $<.0001$ & $\begin{array}{l}28,950 \\
(67.0 \%)\end{array}$ & $4579(71.2 \%)$ & $3401(66.8 \%)$ & $1253(56.6 \%)$ & $<.0001$ \\
\hline Nitrates, \% & $\begin{array}{l}8435 \\
(20.5 \%)\end{array}$ & $1426(23.3 \%)$ & $1935(19.7 \%)$ & 397 (17.5\%) & $<.0001$ & $\begin{array}{l}7164 \\
(16.6 \%)\end{array}$ & $1221(19.0 \%)$ & $830(16.3 \%)$ & $274(12.4 \%)$ & $<.0001$ \\
\hline Loop Diuretic, \% & $\begin{array}{l}20,508 \\
(49.9 \%)\end{array}$ & $\begin{array}{l}2847 \\
(48.25 \%)\end{array}$ & $2394(50.4 \%)$ & $1287(56.7 \%)$ & $<.0001$ & $\begin{array}{l}18,575 \\
(43.0 \%)\end{array}$ & $2642(41.1 \%)$ & $2243(44.0 \%)$ & $962(43.5 \%)$ & 0.0084 \\
\hline Thiazides, \% & $\begin{array}{l}5838 \\
(14.2 \%)\end{array}$ & $939(15.4 \%)$ & $666(14.0 \%)$ & $323(14.2 \%)$ & 0.1137 & $\begin{array}{l}4670 \\
(10.8 \%)\end{array}$ & $785(12.2 \%)$ & $540(10.6 \%)$ & 2055 (9.3\%) & 0.005 \\
\hline Anticoagulant, \% & $\begin{array}{l}4017 \\
(9.8 \%)\end{array}$ & 639 (10.4\%) & $456(9.6 \%)$ & 241 (10.6\%) & 0.1794 & $\begin{array}{l}3715 \\
(8.6 \%)\end{array}$ & 644 (10.0\%) & 407 (8.0\%) & 189 (8.5\%) & $<.0001$ \\
\hline Digoxin, \% & $\begin{array}{l}4410 \\
(10.7 \%)\end{array}$ & $598(9.8 \%)$ & $518(10.9 \%)$ & 287 (12.6\%) & 0.0105 & $\begin{array}{l}4176 \\
(9.7 \%)\end{array}$ & 507 (7.9\%) & $511(10.0 \%)$ & $234(10.6 \%)$ & $<.0001$ \\
\hline
\end{tabular}

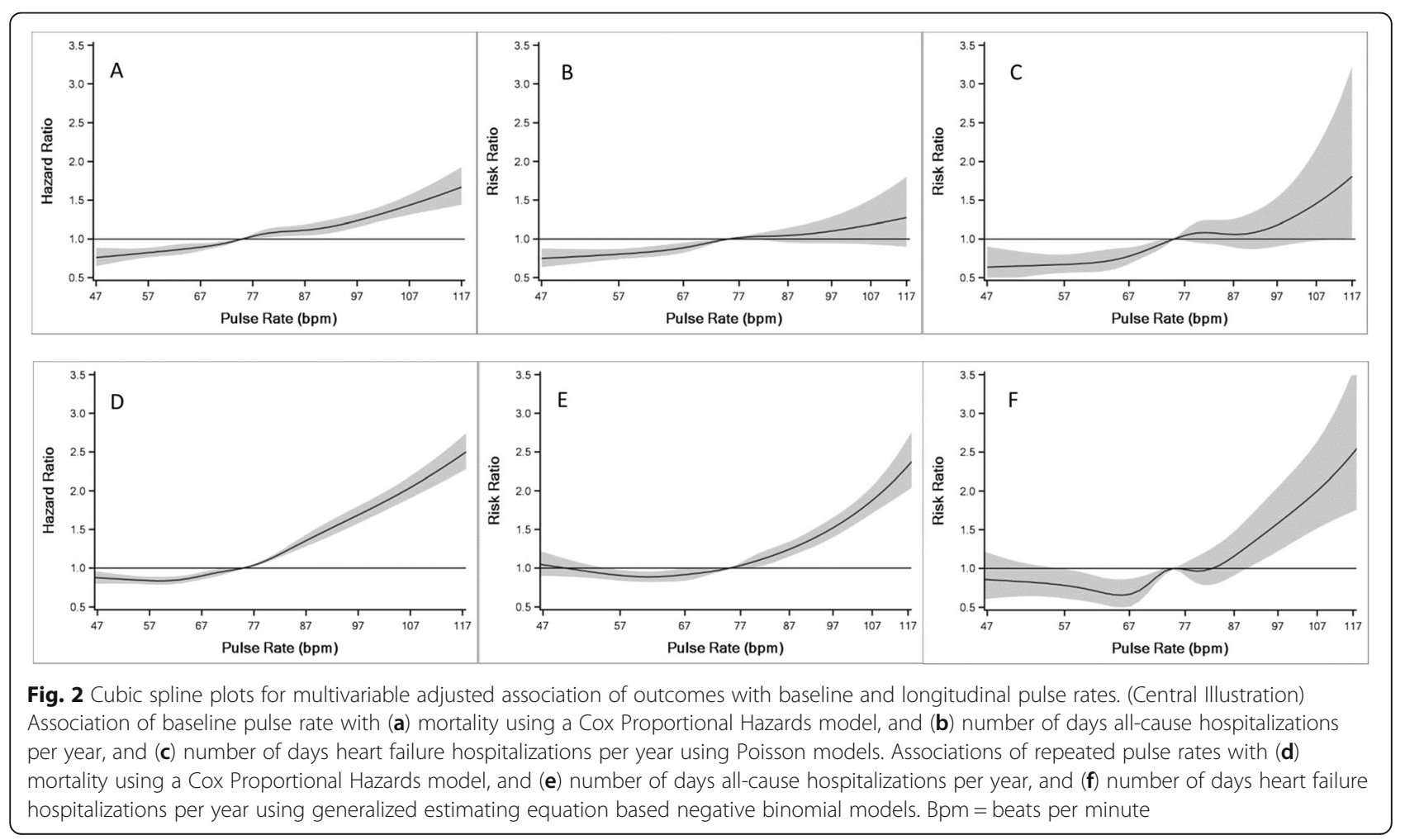


Table 3 Effect of Baseline and Longitudinal Pulse Rates on Outcomes

\begin{tabular}{|c|c|c|c|c|c|}
\hline & Pulse rate (bpm) & $\begin{array}{l}\text { Baseline } \\
\text { Crude } \\
\text { HR or RR }{ }^{a}(95 \% \text { Cl) }\end{array}$ & $\begin{array}{l}\text { Baseline Multivariable } \\
\text { HR or } \operatorname{RR}^{\mathrm{a}}(95 \% \mathrm{Cl})\end{array}$ & $\begin{array}{l}\text { Longitudinal } \\
\text { Crude } \\
\text { HR or RR }{ }^{b}(95 \% \text { Cl) }\end{array}$ & $\begin{array}{l}\text { Longitudinal Multivariable } \\
\text { HR or } \operatorname{RR}^{\mathrm{b}}(95 \% \mathrm{Cl})\end{array}$ \\
\hline \multirow[t]{10}{*}{ All-Cause Mortality (HR) } & $47-59$ & $0.91(0.84-0.98)^{\S}$ & $0.85(0.76-0.94)^{\S}$ & $0.92(0.86-0.98)^{\S}$ & $0.84(0.78-0.90) \|$ \\
\hline & $60-63$ & $0.95(0.88-1.02)$ & $0.90(0.81-1.00)$ & $0.93(0.87-0.99)^{\S}$ & $0.86(0.80-0.94)^{\S}$ \\
\hline & $64-67$ & $0.99(0.92-1.07)$ & $0.92(0.83-1.02)$ & $0.92(0.86-0.98)^{\S}$ & $0.88(0.81-0.95)^{\S}$ \\
\hline & $68-71$ & $0.99(0.92-1.07)$ & $1.01(0.92-1.12)$ & $0.96(0.90-1.02)$ & $0.90(0.83-0.97)^{\S}$ \\
\hline & $72-75$ & - & - & - & - \\
\hline & $76-79$ & $1.10(1.02-1.18)^{\S}$ & $1.11(1.01-1.22)^{\S}$ & $1.07(0.99-1.14)$ & $1.09(1.00-1.18)$ \\
\hline & $80-83$ & $1.07(1.00-1.16)$ & $1.14(1.04-1.27)^{\S}$ & $1.10(1.02-1.18)^{\S}$ & $1.11(1.02-1.21)^{\S}$ \\
\hline & $84-88$ & $1.06(0.99-1.15)$ & $1.15(1.04-1.27)^{\S}$ & $1.19(1.11-1.28) \|$ & $1.22(1.12-1.33) \|$ \\
\hline & $89-96$ & $1.13(1.05-1.22)^{\S}$ & $1.25(1.14-1.38) \|$ & $1.41(1.32-1.52) \|$ & $1.55(1.42-1.68) \|$ \\
\hline & $97-117$ & $1.21(1.12-1.30) \|$ & $1.43(1.30-1.58) \|$ & $1.84(1.71-1.97) \|$ & $2.03(1.86-2.21)^{\|}$ \\
\hline \multirow[t]{10}{*}{ All-Cause Hospitalizations (RR) } & $47-59$ & $0.79(0.70-0.90)^{\S}$ & $0.84(0.73-0.97)^{\S}$ & $0.95(0.87-1.02)$ & $1.00(0.90-1.10)$ \\
\hline & $60-63$ & $0.91(0.80-1.03)$ & $0.98(0.85-1.13)$ & $0.91(0.84-0.99)^{\S}$ & $0.93(0.85-1.03)$ \\
\hline & $64-67$ & $0.92(0.81-1.04)$ & $0.96(0.84-1.10)$ & $0.90(0.83-0.98)^{\S}$ & $0.89(0.81-0.98)^{\S}$ \\
\hline & $68-71$ & $0.94(0.84-1.06)$ & $1.02(0.90-1.16)$ & $0.96(0.89-1.04)$ & $1.02(0.92-1.13)$ \\
\hline & $72-75$ & - & - & - & - \\
\hline & $76-79$ & $1.12(0.99-1.26)$ & $1.16(1.02-1.31)^{\S}$ & $1.01(0.93-1.09)$ & $1.06(0.95-1.18)$ \\
\hline & $80-83$ & $1.21(1.08-1.36) \|$ & $1.19(1.04-1.35)^{\S}$ & $1.17(1.07-1.28)^{\S}$ & $1.22(1.09-1.37)^{\S}$ \\
\hline & $84-88$ & $1.24(1.10-1.39) \|$ & $1.18(1.04-1.34)^{\S}$ & $1.18(1.09-1.29) \|$ & $1.19(1.06-1.34)^{\S}$ \\
\hline & $89-96$ & $1.28(1.14-1.44) \|$ & $1.11(0.98-1.26)$ & $1.44(1.32-1.56) \|$ & $1.43(1.29-1.59) \|$ \\
\hline & $97-117$ & $1.48(1.32-1.65) \|$ & $1.30(1.14-1.47) \|$ & $2.06(1.89-2.25) \|$ & $1.85(1.66-2.07) \|$ \\
\hline \multirow[t]{10}{*}{ Heart Failure Hospitalizations (RR) } & $47-59$ & $0.68(0.49-0.93)^{\S}$ & $0.68(0.48-0.97)^{\S}$ & $0.79(0.57-1.08)$ & $0.88(0.66-1.17)$ \\
\hline & $60-63$ & $0.77(0.56-1.06)$ & $0.87(0.62-1.23)$ & $0.84(0.59-1.19)$ & $0.97(0.70-1.36)$ \\
\hline & $64-67$ & $0.72(0.52-0.99)^{\S}$ & $0.73(0.52-1.03)$ & $0.65(0.47-0.89)^{\S}$ & $0.66(0.50-0.86)^{\S}$ \\
\hline & $68-71$ & $0.96(0.73-1.28)$ & $1.02(0.76-1.38)$ & $0.86(0.59-1.26)$ & $0.98(0.72-1.33)$ \\
\hline & $72-75$ & - & - & - & - \\
\hline & $76-79$ & $1.18(0.89-1.56)$ & $1.23(0.91-1.64)$ & $0.92(0.65-1.30)$ & $1.19(0.81-1.76)$ \\
\hline & $80-83$ & $1.27(0.97-1.68)$ & $1.20(0.89-1.62)$ & $1.05(0.75-1.45)$ & $1.20(0.90-1.61)$ \\
\hline & $84-88$ & $1.38(1.06-1.80)^{\S}$ & $1.23(0.92-1.64)$ & $1.06(0.76-1.46)$ & $1.24(0.92-1.68)$ \\
\hline & $89-96$ & $1.33(1.01-1.74)^{\S}$ & $1.09(0.81-1.46)$ & $1.38(1.00-1.92)$ & $1.61(1.18-2.21)^{\S}$ \\
\hline & $97-117$ & $1.85(1.43-2.39) \|$ & $1.64(1.24-2.17)^{\S}$ & $2.11(1.53-2.92) \|$ & $2.29(1.65-3.17) \|$ \\
\hline
\end{tabular}

HR Hazard Ratio, RR Rate Ratio, bpm beats per minute

aPoisson regression model

${ }^{\mathrm{b}}$ Negative Binomial regression model

'Multivariable models were adjusted for age, gender, race, BMI, eGFR, serum potassium, serum sodium, use of beta blockade, ARBs, ACEls, nitrates, statins, aldosterone antagonists, calcium channel blockers, loop diuretics, and history of coronary artery disease, hypertension, hyperlipidemia, chronic obstructive pulmonary disease, dementia, diabetes, stroke/TIA, cardiovascular disease and anemia. The baseline multivariate model was additionally adjusted for baseline LVEF and the longitudinal multivariate was adjusted for development of atrial fibrillation or atrial flutter

$\S p<.05 \| p<.0001$

survival than those at $<50 \%$ beta blocker target dose $(p=0.0329)$.

\section{Discussion}

Our study, which examined the associations of pulse rate measured from the time of index diagnosis of HFrEF in a large, national cohort, demonstrated a significant association of both baseline pulse rate and pulse rate measured repeatedly over follow-up with morbidity and mortality in heart failure. Our study also demonstrated that guideline directed beta-blocker use was sub-optimal in a real world setting and that higher heart rate was associated with adverse outcomes independent of beta blocker use.

Similar to previous studies [19-23], we found that patients in higher pulse categories were younger, had lower 

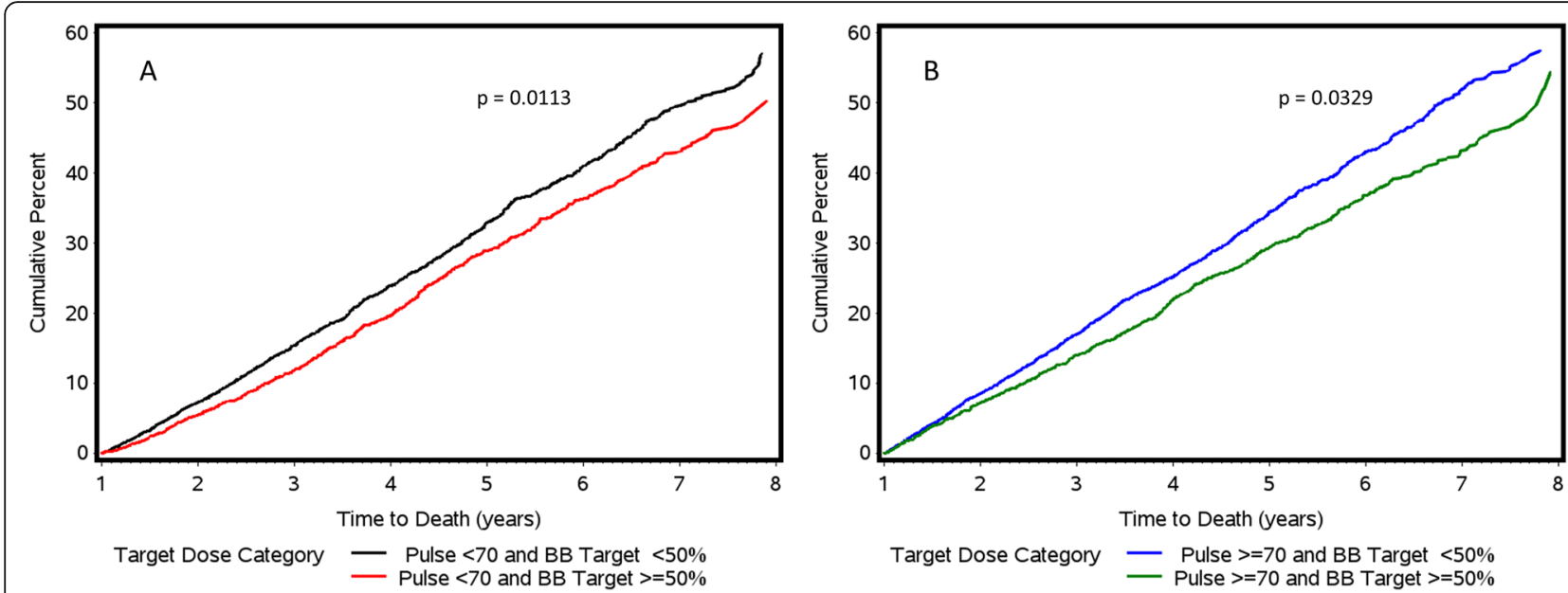

Fig. 3 Cumulative mortality by beta blocker target dose for (a) pulse $<70,(\mathbf{b}) \geq 70 \mathrm{bpm}$. Beta-blocker target dose was assessed 1 year after HFrEF diagnosis and analyses are based on multivariable adjusted Cox proportional hazards models. bb = beta blocker; HFrEF=Heart Failure with Reduced Ejection Fraction; bpm = beats per minute

baseline LVEF values, lower beta blocker use, and higher use of loop diuretics and aldosterone antagonists. The prognostic value of a single heart rate measurement at the time of heart failure diagnosis is not well-known; in our study, baseline pulse rate was positively associated with mortality and hospitalizations. A possible reason is that a high heart rate may be associated with greater neurohormonal activation and thus worse outcomes independent of subsequent treatment. It is also possible that this association is the result of a lack of intensification of therapy with neurohormonal antagonists in the real-world setting, since the proportion of patients receiving therapy did not increase significantly during follow-up, similar to recent reports [24]. Sub-analyses from the Candesartan in Heart Failure-Assessment of Reduction in Mortality and Morbidity (CHARM) trial, placebo arm of the SHIFT trial, and pooled data from the SHIFT and Ivabradine for patients with coronary artery disease and left-ventricular systolic dysfunction (BEAUTIFUL) trials also showed that as baseline heart rate (albeit at time of entry into clinical trial and not at index diagnosis of HFrEF) increased, there was a significant increased risk of all-cause mortality, as well as the composite outcome of cardiovascular death or HF hospitalization $[15,19,25]$. Similarly, a meta-analysis of 11 randomized controlled trials of beta blockers in HF patients in sinus rhythm showed a significant positive linear association between heart rate at time of enrollment and all-cause mortality [22]. Also in accordance with our findings, Ibrahim et al., who examined associations of heart rate in a single center cohort from baseline (first visit where patients had $\mathrm{LVEF} \leq 35 \%$ and sinus rhythm), demonstrated a positive association between baseline heart rate and all-cause mortality or HF hospitalization [21]. However, a post-hoc analysis from the Efficacy of Vasopressin Antagonism in Heart Failure Outcome Study with Tolvaptan (EVEREST) trial showed that baseline heart rate was not significantly associated with all-cause mortality $(p \geq 0.066)$ in HFrEF patients in sinus rhythm [20]. The results of our study in a large U.S. real-world population, conclusively demonstrate the predictive value of pulse rate measured at the time of diagnosis of HFrEF. In the meta-analysis of clinical trials described above, heart rates at interim visits (mean 184 days after randomization and unknown time relative to diagnosis) were more predictive of mortality than change in heart rate from randomization [22]. Greene et al., in their post-hoc analysis of the EVEREST trial mentioned above [20], found that heart rates $\geq 70 \mathrm{bpm}$ postdischarge (only through 4 weeks) were associated with increased risk of mortality. Similar to our serial measurement approach, Hamill and colleagues studied the effect of heart rate at time of entry into clinical trial and of repeated heart rate measurements, albeit only in the year following enrollment [25]. Their cohort only included 8, 699 patients, but still found a strong association between baseline heart rate and HF hospitalization and cardiovascular death, with associations strengthened for repeated heart rate measurements. Our study was much larger, including only HFrEF patients, and had a longer median follow-up period of 3.2 years from a true baseline timepoint.

Most studies that have looked at the association between heart rate and outcomes were post-hoc analyses of clinical trials. Patients were only eligible to enroll in many trials if they had a heart rate $\geq 60 \mathrm{bpm}$ or $\geq 70 \mathrm{bpm}$ $[23,25,26]$, while in our real-world study we examined patients with pulse rates as low as $47 \mathrm{bpm}$. In previous 
studies, repeated heart rate measurements were obtained only during study mandated visits often limited to one follow-up visit within the first year after study enrollment $[20,22,25]$, while we had an average of $6.3 \pm 3.6$ pulse measurements per patient updated at 6 month intervals over a median follow-up of 3.2 years. Previous studies except one single-center observational cohort study [21] also defined baseline as the time of randomization and enrollment into the clinical trial, which is unlikely to have been a true "baseline" at the time of index diagnosis.

Although multiple studies have shown that heart rate reduction is associated with improved clinical outcomes in heart failure patients [26-31], it remains controversial whether benefits from beta blockers are stronger due to achievement of target doses that help to reduce heart rate [32] or from the magnitude of heart rate reduction $[27-29,33]$. In our study, only $15 \%$ achieved $\geq 50 \%$ target dose of guideline directed beta blockade. Similar to other studies [21, 22], our study also demonstrated that a lower pulse rate is associated with better outcomes in HFrEF independent of treatment with beta-blockade. However, in patients who were on guideline directed beta blockers, our results suggest that achieving at least $50 \%$ of target dose was also an important predictor of better outcomes at both low and high pulse rates. While both lower heart rate and higher beta blocker target dose are associated with outcomes in heart failure patients, further studies are necessary to better understand their roles together in improving outcomes. Although we were unable to assess the effect of ivabradine in our cohort, it warrants further investigation into how effectively it improves heart failure outcomes through reduction of heart rate in real world settings relative to or in combination with beta-blockers [15, 34].

\section{Strengths and limitations}

There are several major strengths of this real-world study. This study was done in a large national cohort; baseline pulse rates close to the index diagnosis of HFrEF were used for analyses; serial pulse rates and three major outcomes were measured over a long period of follow-up; and electronic health records were mined to obtain detailed and serial clinical, laboratory, and medication data. Inclusion of a significant number of African Americans also lends strength to this study. However, there were some limitations. Since this was a retrospective cohort study, there remains potential for unmeasured confounding after model adjustment. To minimize the potential for bias in retrospective studies, the study population and exposure categories were systematically defined, and we captured outcomes after diagnosis using data from electronic medical records. There was potential for differential misclassification since patients who were sicker likely received more medical care and thus had more pulse measurements, however a single measurement was observed at baseline and all longitudinal pulse rates were updated at regular 6month intervals allowing patients to oscillate between categories and carrying forward any value recorded in the prior year. As expected in a VA cohort, there was a high proportion of white males, however we adjust for both race and gender in our analyses. To account for care received outside the VA system, we utilized data from CMS; though it is possible that we may not have captured the entirety of care received outside the VA.

\section{Conclusions}

A lower pulse rate at the time of HFrEF diagnosis and across follow-up clinical encounters is strongly associated with lower risk of mortality and hospitalization outcomes in a real-world setting, independent of the use of beta blockade. Strategies to lower heart rate, including use of beta blockade, may be beneficial in reducing the morbidity and mortality associated with HFrEF.

\section{Supplementary information}

Supplementary information accompanies this paper at https://doi.org/10. 1186/s12872-020-01384-6.

Additional file 1. Contains additional information regarding the

methods used.

\section{Abbreviations}

ACEl: Angiotensin converting enzyme inhibitors; ARB: Angiotensin receptor blockers; BEAUTIFUL: Ivabradine for patients with coronary artery disease and left-ventricular systolic dysfunction trial; BMI: Body mass index; BPM: Beats per minute; CHARM: Candesartan in heart failure-assessment of reduction in mortality and morbidity trial; Cl: Confidence interval; CMS: Centers for Medicare \& Medicaid; CPT: Current procedural terminology; eGFR: Estimated glomerular filtration rate; EVEREST: Efficacy of vasopressin antagonism in heart failure outcome study with Tolvaptan trial; GEE: Generalized estimating equation; HFrEF: Heart failure with reduced ejection fraction; HR: Hazard ratio; ICD-9: International classification of disease ninth revision; LVEF: Left ventricular ejection fraction; NLP: Natural language processing; RR: Rate ratio; SHIFT: Ivabradine and outcomes in chronic heart failure; TIA: Transient ischemic attack; TIU: Text integration utilities; VA: Veterans affairs

\section{Acknowledgements \\ The authors are grateful to Ms. Constance Nelson for her assistance with this project. Support for VA/CMS data provided by the Department of Veterans Affairs, VA Health Services Research and Development Service, VA Information Resource Center (Project Numbers SDR 02-237 and 98-004).}

\section{Authors' contributions}

KEK: designed the study, oversaw data analysis and drafted the manuscript. PS, RP: extracted the data, performed the data analysis and critically reviewed and edited the manuscript for content; JBR: reviewed and edited the manuscript for content; LD, DRG: designed the study, oversaw the data analysis, reviewed and edited the manuscript for the content; and JJ: designed and supervised the study, received funding, reviewed and edited the manuscript for content. The author(s) read and approved the final manuscript. 


\section{Funding}

This project was supported by a research grant from Amgen Inc. to Dr. Joseph. Amgen did not have any role in the collection, analysis or interpretation of the data, but did review the manuscript for content.

\section{Availability of data and materials}

The data that support the findings of this study are available from the United States Department of Veterans Affairs corporate data warehouse, but since this project was not federally funded and in accordance with current VA policies, restrictions apply to the availability of these data, which were used for the current study, and so are not publicly available. Methods used in analysis, however, may be provided to any researcher for purposes of replicating the procedure upon request from the senior author.

\section{Ethics approval and consent to participate}

This study was approved by the VA Boston Healthcare System Institutional Review Board (IRB\#3042). Given that this was a database study, informed consent was not required. Access to raw data from the United States Department of Veterans Affairs corporate data warehouse was granted by the VA Boston Healthcare System Institutional Review Board and the Veterans Health Administration National Data Systems. Access to the Center for Medicare and Medicaid data was granted through the VA Information resource Center in accordance with VHA Directive 1153 and VHA Handbook 1153.01

\section{Consent for publication}

Not applicable.

\section{Competing interests}

The authors declare that they have no competing interests.

\section{Author details}

'Massachusetts Veterans Epidemiology and Research Information Center (MAVERIC), Veterans Affairs Boston Healthcare System, Boston, MA, USA. ${ }^{2}$ Department of Medicine, Division of Aging, Brigham and Women's Hospital, Harvard Medical School, Boston, MA, USA. ${ }^{3}$ Amgen Inc., Thousand Oaks, CA, USA. ${ }^{4}$ Department of Biostatistics, Boston University School of Public Health Boston, MA, USA. ${ }^{5}$ Department of Medicine, Division of Cardiovascular Medicine, Brigham and Women's Hospital, Harvard Medical School, Boston, MA, USA. ${ }^{6}$ Cardiology Section, VA Boston Healthcare System, 1400 VFW Parkway, West Roxbury, MA 02132, USA.

\section{Received: 3 December 2019 Accepted: 10 February 2020}

\section{Published online: 26 February 2020}

\section{References}

1. Basuray A, French B, Ky B, Vorovich E, Olt C, Sweitzer NK, et al. Heart failure with recovered ejection fraction: clinical description, biomarkers, and outcomes. Circulation. 2014;129(23):2380-7.

2. Benjamin EJ, Blaha MJ, Chiuve SE, Cushman M, Das SR, Deo R, et al. Heart disease and stroke Statistics-2017 update: a report from the American Heart Association. Circulation. 2017;135(10):e146-603.

3. Jensen MT, Marott JL, Allin KH, Nordestgaard BG, Jensen GB. Resting heart rate is associated with cardiovascular and all-cause mortality after adjusting for inflammatory markers: the Copenhagen City heart study. Eur J Prev Cardiol. 2012;19(1):102-8

4. Reil JC, Reil GH, Bohm M. Heart rate reduction by If-Channel inhibition and its potential role in heart failure with reduced and preserved ejection fraction. TCM. 2009;19(5):152-7.

5. Fox K, Borer JS, Camm AJ, Danchin N, Ferrari R, Lopez Sendon JL, et al. Resting heart rate in cardiovascular disease. J Am Coll Cardiol. 2007;50(9): 823-30.

6. Kannel WB, Kannel C, Paffenbarger RS, Cupples LA. Heart rate and cardiovascular mortality: the Framingham study. Am Heart J. 1987;113(6): 1489-94.

7. Palatini $P$, Julius $S$. Elevated heart rate: a major risk factor for cardiovascular disease. Clin Exp Hypertens. 2004;26(7-8):637-44

8. Diaz A, Bourassa MG, Guertin MC, Tardif JC. Long-term prognostic value of resting heart rate in patients with suspected or proven coronary artery disease. Eur Heart J. 2005;26(10):967-74
9. Fox K, Ford I, Steg PG, Tendera M, Robertson M, Ferrari R. Heart rate as a prognostic risk factor in patients with coronary artery disease and left ventricular systolic dysfunction (BEAUTIFUL): a subgroup analysis of a randomised controlled trial. Lancet. 2008;372(9641):817-21.

10. Ho JE, Bittner V, Demicco DA, Breazna A, Deedwania PC, Waters DD. Usefulness of heart rate at rest as a predictor of mortality, hospitalization for heart failure, myocardial infarction, and stroke in patients with stable coronary heart disease (data from the treating to new targets [TNT] trial). Am J Cardiol. 2010;105(7):905-11.

11. Kolloch R, Legler UF, Champion A, Cooper-Dehoff RM, Handberg E, Zhou Q, et al. Impact of resting heart rate on outcomes in hypertensive patients with coronary artery disease: findings from the INternational VErapamil-SR/ trandolapril STudy (INVEST). Eur Heart J. 2008:29(10):1327-34.

12. Pocock SJ, Wang D, Pfeffer MA, Yusuf S, McMurray JJ, Swedberg KB, et al. Predictors of mortality and morbidity in patients with chronic heart failure. Eur Heart J. 2006;27(1):65-75.

13. Hillis GS, Woodward M, Rodgers A, Chow CK, Li Q, Zoungas S, et al. Resting heart rate and the risk of death and cardiovascular complications in patients with type 2 diabetes mellitus. Diabetologia. 2012;55(5):1283-90.

14. Reil JC, Custodis F, Swedberg K, Komajda M, Borer JS, Ford I, et al. Heart rate reduction in cardiovascular disease and therapy. Clin Res Cardiol. 2011; 100(1):11-9.

15. Böhm M, Swedberg K, Komajda M, Borer JS, Ford I, Dubost-Brama A, et al. Heart rate as a risk factor in chronic heart failure (SHIFT): the association between heart rate and outcomes in a randomised placebo-controlled trial. Lancet. 2010:376(9744):886-94.

16. US Department of Veterans Affairs. System of Records Notice 97VA10P1: Consolidated Data Information System-VA. 76 FR 25409. 2013.

17. Yancy CW, Jessup M, Bozkurt B, Butler J, Casey DE Jr, Colvin MM, et al. 2017 ACC/AHA/HFSA focused update of the 2013 ACCF/AHA guideline for the Management of Heart Failure: a report of the American College of Cardiology/American Heart Association task force on clinical practice guidelines and the Heart Failure Society of America. J Am Coll Cardiol. 2017 70(6):776-803.

18. Thomas L, Reyes EM. Tutorial: survival estimation for cox regression models with time-varying Coe cients using SAS and R. J Stat Softw. 2014:61:1-23.

19. Castagno D, Skali H, Takeuchi M, Swedberg K, Yusuf S, Granger CB, et al. Association of heart rate and outcomes in a broad spectrum of patients with chronic heart failure: results from the CHARM (candesartan in heart failure: assessment of reduction in mortality and morbidity) program. J Am Coll Cardiol. 2012;59(20):1785-95.

20. Greene SJ, Vaduganathan M, Wilcox JE, Harinstein ME, Maggioni AP, Subacius $H$, et al. The prognostic significance of heart rate in patients hospitalized for heart failure with reduced ejection fraction in sinus rhythm: insights from the EVEREST (efficacy of vasopressin antagonism in heart failure: outcome study with Tolvaptan) trial. JACC Heart Fail. 2013;1 (6):48896.

21. Ibrahim NE, Gaggin HK, Turchin A, Patel HK, Song Y, Trebnick A, et al. Heart rate, beta-blocker use, and outcomes of heart failure with reduced ejection fraction. Eur Heart J Cardiovasc Pharmacother. 2019;5(1):3-11.

22. Kotecha D, Flather MD, Altman DG, Holmes J, Rosano G, Wikstrand J, et al. Heart rate and rhythm and the benefit of Beta-blockers in patients with heart failure. J Am Coll Cardiol. 2017:69(24):2885-96.

23. Bohm M, Borer J, Ford I, Gonzalez-Juanatey JR, Komajda M, Lopez-Sendon J, et al. Heart rate at baseline influences the effect of ivabradine on cardiovascular outcomes in chronic heart failure: analysis from the SHIFT study. Clin Res Cardiol. 2013;102(1):11-22

24. Greene SJ, Butler J, Albert NM, DeVore AD, Sharma PP, Duffy Cl, et al. Medical therapy for heart failure with reduced ejection fraction: the CHAMP-HF registry. J Am Coll Cardiol. 2018;72(4):351-66.

25. Hamill V, Ford I, Fox K, Bohm M, Borer JS, Ferrari R, et al. Repeated heart rate measurement and cardiovascular outcomes in left ventricular systolic dysfunction. Am J Med. 2015;128(10):1102-8 e6.

26. Swedberg K, Komajda M, Böhm M, Borer JS, Ford I, Dubost-Brama A, et al. Ivabradine and outcomes in chronic heart failure (SHIFT): a randomised placebo-controlled study. Lancet. 2010;376(9744):875-85.

27. Cullington D, Goode KM, Clark AL, Cleland JG. Heart rate achieved or betablocker dose in patients with chronic heart failure: which is the better target? Eur J Heart Fail. 2012;14(7):737-47.

28. Flannery G, Gehrig-Mills R, Billah B, Krum H. Analysis of randomized controlled trials on the effect of magnitude of heart rate reduction on 
clinical outcomes in patients with systolic chronic heart failure receiving beta-blockers. Am J Cardiol. 2008;101(6):865-9.

29. McAllister FA, Wiebe N, Ezekowitz JA, Leung AA, Armstrong PW. Metaanalysis: Beta-Blocker Dose, Heart Rate Reduction, and Death in Patients With Heart Failure. Ann Intern Med. 2009;150:784-94.

30. Porapakkham P, Porapakkham P, Krum H. Is target dose of beta-blocker more important than achieved heart rate or heart rate change in patients with systolic chronic heart failure? Cardiovasc Ther. 2010;28(2):93-100.

31. Swedberg K. Pure heart rate reduction: further perspectives in heart failure. Eur Heart J Supplements. 2007;9(suppl_F):F20-F4.

32. Fiuzat M, Wojdyla D, Pina I, Adams K, Whellan D, O'Connor CM. Heart rate or Beta-blocker dose? Association with outcomes in ambulatory heart failure patients with systolic dysfunction: results from the HF-ACTION trial. JACC Heart Fail. 2016;4(2):109-15.

33. Swedberg K, Komajda M, Bohm M, Borer J, Robertson M, Tavazzi L, et al. Effects on outcomes of heart rate reduction by ivabradine in patients with congestive heart failure: is there an influence of beta-blocker dose?: findings from the SHIFT (systolic heart failure treatment with the I(f) inhibitor ivabradine trial) study. J Am Coll Cardiol. 2012;59(22):1938-45.

34. Scicchitano P, Cortese F, Ricci G, Carbonara S, Moncelli M, lacoviello M, et al. Ivabradine, coronary artery disease, and heart failure: beyond rhythm control. Drug Des Devel Ther. 2014:8:689-700.

\section{Publisher's Note}

Springer Nature remains neutral with regard to jurisdictional claims in published maps and institutional affiliations.

Ready to submit your research? Choose BMC and benefit from:

- fast, convenient online submission

- thorough peer review by experienced researchers in your field

- rapid publication on acceptance

- support for research data, including large and complex data types

- gold Open Access which fosters wider collaboration and increased citations

- maximum visibility for your research: over $100 \mathrm{M}$ website views per year

At BMC, research is always in progress.

Learn more biomedcentral.com/submissions 